\title{
Pension fund management with investment certificates and stochastic dominance
}

\author{
Sebastiano Vitali ${ }^{1,2}$. Vittorio Moriggia ${ }^{2}$ \\ Accepted: 30 October 2020 / Published online: 9 November 2020 \\ (c) The Author(s) 2020
}

\begin{abstract}
This paper considers an extension of the common asset universe of a pension fund to investment certificates. Investment certificates are a class of structured products particularly interesting for their special payoff structures and they are acquiring relevancy in the worldwide markets. In fact, some subclasses of certificates offer loss protection and show high liquidity and, thus, they can be very appreciated by pension fund managers. We consider the problem of a pension fund manager who has to implement an Asset and Liability Management model trying to achieve a long-term sustainability. Therefore, we formulate a multi-stage stochastic programming problem adopting a discrete scenario tree and a multi-objective function. We propose a technique to price highly structured products such as investment certificates on a discrete scenario tree. Finally, we solve the investment problem considering some investment certificate types both in term of payoff structure and protection level, and we test whether they are preferred or not to standard hedging contract such as put options. Moreover, we test the inclusion of first-order and second-order stochastic dominance constraints on multiple stages with respect to a benchmark portfolio. Numerical results show that the portfolio composition reacts to the inclusion of the stochastic dominance constraints, and that the optimal portfolio is efficiently able to reach several targets such as liquidity, returns, sponsor's extraordinary contribution and funding gap.
\end{abstract}

Keywords Stochastic programming · Portfolio selection - Sensitivity analysis · Asset and liability management $\cdot$ Pension fund $\cdot$ Investment certificates $\cdot$ Discrete pricing

The research of Sebastiano Vitali was supported by Czech Science Foundation (Grant 19-28231X) and by MIUR-ex60\% 2020 sci.resp. Sebastiano Vitali. The research of Vittorio Moriggia was supported by MIUR-ex60\% 2019 sci.resp. Vittorio Moriggia and by MIUR-ex60\% 2020 sci.resp. Vittorio Moriggia.

$凶$ Sebastiano Vitali

sebastiano.vitali@unibg.it

Vittorio Moriggia

vittorio.moriggia@unibg.it

1 Faculty of Mathematics and Physics, Department of Probability and Mathematical Statistics, Charles University, Sokolovska 83, 18675 Prague, Czech Republic

2 Department of Management, Economics and Quantitative Methods, University of Bergamo, Via dei Caniana 2, 24127 Bergamo, Italy 
Mathematics Subject Classification 90C15 $\cdot 90 \mathrm{C} 29 \cdot 91 \mathrm{~B} 28 \cdot 91 \mathrm{~B} 30$

\section{Introduction}

Pension funds must be managed investing the assets that the pensioners paid during their working life and ensuring the payments of the pensions. Thus, a pension fund management can be addressed via Asset and Liability Management (ALM) models which have been deeply explored in last fifty years. The introduction to ALM models for the management of an investing portfolio started with the BONDS model proposed in Bradley and Crane (1972), Bradley and Crane (1980). Still, for a deeper analysis of the liability side, we have to wait until the MIDAS model in Dempster and Ireland (1988), Dempster and Ireland (1989), Dempster and Ireland (1991), and other models such as in Kusy and Ziemba (1986). Then, it appeared natural to adopt a multistage stochastic formulation to tackle the uncertainty that characterises such type of problem. The milestone Russell-Yasuda Kasai Model described in Cariño et al. (1994) and then analysed in Cariño and Ziemba (1998a) and Cariño et al. (1998b) developed an ALM within a multistage stochastic framework. In the same years other models and analysis have been proposed, see e.g. Mulvey (1994a), Mulvey (1994b), Nielsen and Zenios (1996) and Zenios (1995), among which the Towers Perrin-Tillinghast ALM model and the Tower Perrin scenario generation system had a great relevance, see (Mulvey et al. 2000).

ALM models can be formulated to face the specificity of a pension fund. For instance, Pflug and Świetanowski (1999) proposed a ALM model for pension funds paying a particular attention to both the asset and the liability side modelling. Consigli and Dempster (1998a), Consigli and Dempster (1998b) introduced the CALM model which handle the pension fund management problem considering a long-term objective and different types of pension contracts. A similar approach appeared in Dempster et al. (2003).

The two main features that characterize a pension fund are: the defined benefit or defined contribution schema, and the pillar the pension fund belongs to. In a defined contribution pension fund the final pension benefit is unknown at the beginning and it will be the results of the contribution investment, i.e. the pensioner bears the risk; in a defined benefit pension fund the final benefit is known since the beginning of the pension contract, i.e. the pension fund sponsor bears the risk. A specific focus on the defined benefit pension fund can be found in Dert (1998). The second distinction relies on the considered pension pillar. There exist three pension pillars. The first is the State Pension system, the second is based on the worker category and/or on the employee's employer, the third is composed of private insurance contracts. For instance, the InnoALM model proposed in Ziemba (2007) and Geyer and Ziemba (2008) considers a second pillar pension fund since it is built to manage the pension fund of an electricity company. Our work develops within the defined benefit framework and considers a second pillar pension fund.

The suitability of multistage approach to deal with ALM problems has been proved in multiple works during the last decades. Mulvey et al. (2006) suggested a multiperiod model to increase the understanding of risk and reward in a long-term horizon framework for pension plans and other long-term investors, see also (Mulvey et al. 2007, 2008). Comprehensive collections are in Ziemba and Mulvey (1998) and Zenios and Ziemba (2006), Zenios and Ziemba (2007). More recent developments are in Kopa and Rusỳ (2020) that formulated an ALM model to tackle the consumer loan problem; in Consigli et al. (2011), Consigli and di Tria (2012), Consigli and Moriggia (2014), and in Consigli et al. (2017) that analysed 
a Property \& Casualty insurance fund; and in other papers that discussed the pension fund from different point of view such as the issuer of the pension fund, see e.g Vitali et al. (2017) and Devolder et al. (2020), and the individual pension problem, see e.g. Consigli (2007), Consigli et al. (2012), Kopa et al. (2018) and Consigli et al. (2019).

A particular focus have been dedicate to follow the evolution of the asset universe according to regulatory updates, cf. McKendall et al. (1994), Davis (2000), Guiso et al. (2002), Hardy (2003), Gollier (2008), Broeders et al. (2009). Following this path, our aim is to propose an extension of the model proposed in Consigli et al. (2017) and in Moriggia et al. (2019) to include a relatively new class of investment opportunities, i.e. the investment certificates.

Investment certificates are financial instruments that offer a competitive yield and usually include some safety clause regarding the principal amount. These instruments can replicate a wide range of investment strategies and, therefore, they provide to investors a wide variety of risk-return profiles. More specifically, an investment certificate is a securitised derivative instrument issued by an investment bank that replicates the trend of the underlying with the possibility of having a leverage effect, a premium in the case of market stability and/or a protection property. Recently, these products have become particularly attractive to investors, both institutional and retail, for several reasons: their asymmetric payoff, their not long-only behaviour, and, for instance in the Italian system, because they allow a fiscal optimisation of the portfolio. In the contest of a pension fund, following the classification proposed by the European Structured Investment Products Association (EUSIPA) ${ }^{1}$, our purpose is to investigate the inclusion in the asset universe of three types of investment certificates: the capital protection, the yield enhancement, and the participation classes. Indeed, we want to restrict our analysis to those investment certificates that have a protection effect either total or partial. Moreover, to stress the fact that these contracts can be used only for protection purposes, we forbid the selling, i.e. the speculation on their market price: once the products has been bought, it must be kept until its maturity. We assume that in the market there exist investment certificates on the two most risky assets that are also in the asset universe: Public Equity and Real Estate. This hypothesis is needed to test if such products could really be of interest for a pension fund. In this case, also in the real world, the pension fund manager will ask a financial institution to issue such kind of products that will be bought by the pension fund itself at the fair price. Therefore, another issue that this paper is addressing is the pricing of these products considering the discrete scenario tree on which the model is formulated and solved. Moreover, we will compare the results obtained with investment certificates with the results we would obtain using standard hedging contracts such as put option as in Moriggia et al. (2019). In particular, we observe whether the investment certificates can substitute the put options to hedge the most risky portfolio positions. Finally, following Moriggia et al. (2019), we test the inclusion of second-order stochastic dominance constraints with respect to a benchmark portfolio, and, for a more complete analysis, we include also first-order stochastic dominance. The inclusion of stochastic dominance in Economics and Finance goes back to the seminal work of Quirk and Saposnik (1962), and then of Hadar and Russell (1969) and Hanoch and Levy (1969). Later on, the first-order stochastic dominance constraints were applied in stochastic programming problem in Kuosmanen (2004), Dentcheva and Ruszczyński (2004) and Dupačová and Kopa (2014). Similarly, second-order stochastic dominance constraints were used in Dentcheva and Ruszczynski (2003) and Luedtke (2008) and to portfolio efficiency analysis in Post (2003), Kuosmanen (2004), Dupačová and Kopa (2012) and Kopa and Post (2015). In multistage stochastic programming, the second-order stochastic dominance constraints were applied to asset-liability modelling in Yang et al.

1 www.eusipa.org. 
(2010) and in Moriggia et al. (2019), and in an individual pension allocation problem in Kopa et al. (2018) and Consigli et al. (2019).

To summarize, the contributions of this work are:

- to propose how to price highly structured financial products, such as investment certificates, on a discrete scenario tree,

- to observe if the inclusion of three types of investment certificates within an ALM model for pension fund can improve the performance of the portfolio,

- to compare the impact in terms of portfolio protection of the investment certificates with respect to standard put options,

- to study the sensitivity of the investment strategy with respect to stochastic dominance, both first-order and second-order stochastic dominance.

The paper is structured as follows. Section 2 introduces: the formulation of the ALM model, a specific focus on stochastic dominance constraints, the structure of the investment certificate adopted in this work, and the technique to price highly structured products on a discrete scenario tree. Section 3 presents the settings of the model and the definition of the portfolio benchmark. In Sect. 4 we show the results of the model comparing the performance under different settings, we analyse the sensitivity of the portfolio with respect to the structure of the derivatives within the asset universe, and we observe the stability of the stochastic solution with respect to the generated scenario tree. The paper concludes in Sect. 5.

\section{Model description}

Following Consigli et al. (2017), the stochastic tree is differentiated between decisional nodes and intermediate nodes. In the decisional nodes buying and selling are allowed, while in intermediate nodes no multiple branching originates and we account pension payments and portfolio income. The decisional stages are denoted $t_{h}, h=0, \ldots, H$, where $t_{0}=0$ represents the initial stage. The scenario tree is represented with the nodal notation, for more details refer to Consigli et al. (2017). In particular, we denote each node with index $n$, the set of its descendant nodes $C(n)$, while $t(n)$ expresses the stage of node $n$. Each node contains the nodal price returns and the nodal income returns for each asset. With nodal price (income) return we mean the return $\rho_{i, n, \tilde{n}}$ generated by an asset $i$ from a node $n$ to its descendant nodes $\tilde{n} \in C(n)$. Following Moriggia et al. (2019), we generate the nodal price returns and the nodal income returns with a multivariate and hierarchical linear regression, and the nodal returns generated by the put options that will be included in the model for the sake of comparison.

Moving from this approach, for the purpose of the current analysis we need to develop a technique to define the return generated by highly structured products on a discrete scenario tree. As it has been done for the pricing of the put options, also for highly structured products we cannot use a model derived by the classic Black and Scholes (B\&S) formula since we deal with a discrete (and relatively low) amount of possible paths for underlyings that do not follow a Brownian Motion process. However, whereas for the pricing of the put options it is possible to use the payoff at maturity of the option, when we have to compute the return generated by an investment certificate $i$, we need to account its possible values - and not the payoffs - at its maturity $m(i)$. The final value of each investment certificate depends on a complex structure that takes as input the movements of the underlying asset. Assuming that the investment certificate $i$ has as underlying the asset $j$, we define a map $f(\cdot)$ that relates the return $\rho_{j, n, \tilde{n}}$, generated by the underlying from node $n$ and its descendant $\tilde{n} \in C(n)$ with $t(\tilde{n})-t(n)=m(i)$, with the value $p(i, \tilde{n})$ at the maturity of the investment certificate: 


$$
p_{i, \tilde{n}}=f\left(\rho_{j, n, \tilde{n}}\right)
$$

Once we have computed the final value $p_{i, \tilde{n}}$ for every $\tilde{n} \in C(n), t(\tilde{n})-t(n)=m(i)$, we compute the current fair value $p_{i, n}$ of the investment certificate discounting its expected final value with the risk-free interest rate $r_{n, \tilde{n}}$ from node $\tilde{n}$ to $n$ :

$$
p_{i, n}=\mathbb{E}\left[e^{-r_{n, \tilde{n}}[t(\tilde{n})-t(n)]} \cdot p_{i, \tilde{n}}\right]
$$

Finally, the returns produced by the investment certificate are computed by

$$
\rho_{i, n, \tilde{n}}=\frac{p_{i, \tilde{n}}-p_{i, n}}{p_{i, n}}
$$

In this way, knowing the price return for the underlying, we are able to populate the scenario tree with the returns of the investment certificates. As it will be shown in the subsequent section, each investment certificate is defined by a specific map $f(\cdot)$.

Following the suggestion in Consigli et al. (2017), we define a multicriteria objective function that aggregates a short-term risk control, a medium-term profitability and a longterm sustainability. The objective function aims to minimize the expected shortfall of a set $A$ of variables $Y_{a}, a \in A$ with respect to a specific threshold $\bar{Y}_{a}$. The $a$-th variable is accounted only in a specific stage $\tau_{a}$. The expected shortfalls are combined associating a weight $\lambda_{a}$ to each objective variable, with $\sum_{a \in A} \lambda_{a}=1$. Therefore, the objective function is:

$$
\min \left\{\sum_{a \in A} \lambda_{a} \cdot \mathbb{E}\left[\bar{Y}_{a}-Y_{a, n} \mid Y_{a, n}<\bar{Y}_{a}\right], \quad \forall n \mid t(n)=\tau_{a}\right\} .
$$

The other constraints of the ALM model is based on the original model in Consigli et al. (2017) and its modification presented and explained in Moriggia et al. (2019) concerning the liability evaluation, the inclusion of hedging contracts within the selection problem and the first-order stochastic dominance and the second-order stochastic dominance constraints. The detailed definition of the stochastic dominance constraints is specified in the subsequent section.

\subsection{Stochastic dominance constraints}

The stochastic dominance (SD) is a statistical tool that can be used to compare and rank random variables. There exist several definitions of stochastic dominance. Each of them corresponds to a specific class of utility function. In particular, the definition of the first-order stochastic dominance (FSD) relation is as follows: A random variable $A$ FSD dominates a random variable $B$ if the cumulative distribution function of $A$ is below that of $B$. This is equivalent to state that every agent with an increasing utility function prefers $A$ to $B$. Similarly, the definition of the second-order stochastic dominance (SSD) relation is as follows: A random variable $A$ SSD dominates a random variable $B$ if the integrated cumulative distribution function of $A$ is below that of $B$. Again, this is equivalent to state that every agent with an increasing and concave utility function prefers $A$ to $B$. Given these definitions, it is clear that if $A$ FSD dominates $B$, then $A$ SSD dominates $B$, while the opposite is not always true. There exists several other declinations of stochastic dominance, for example the third-order stochastic dominance, see Post and Kopa (2017), and the recent DARA stochastic dominance, see (Post et al. 2015).

Stochastic dominance can be easily checked ex-post, once the distributions of the random variables are known, or can be included in the optimization model as constraints. In our case, 
we want to compare the vector $\mathbf{w}_{t_{h}}$ of the optimal portfolio wealth realizations occurring in all nodes at stage $t_{h}$, with the vector $\mathbf{w}_{t_{h}}^{B}$ of a benchmark portfolio wealth realizations. These random variables are clearly discrete with equiprobable realizations. Thus, it is useful to formulate the FSD and SSD conditions using a double stochastic matrix as proposed in Kuosmanen (2004) including the following constraint:

$$
\mathbf{w}_{t_{h}} \geq \mathbf{Q} \cdot \mathbf{w}_{t_{h}}^{B}
$$

for some matrix $\mathbf{Q}$ which is double stochastic, i.e. it satisfies the following conditions:

$$
\begin{aligned}
& \sum_{i} Q_{i, j}=1 \\
& \sum_{j} Q_{i, j}=1 .
\end{aligned}
$$

Then, if we impose that the elements of $\mathbf{Q}$ are binary, we obtain the FSD; while if we impose that the elements of $\mathbf{Q}$ belong to the interval [0, 1], we obtain the SSD. In the case of SSD, all these constraints are linear and, thus, the model remains, typically, tractable. In the case of FSD, the problem becomes mixed-integer and the computational burden could easily make the problem intractable.

As we will show later on, we include stochastic dominance constraints on two different stages. Thus, the stochastic dominance constraints are imposed simultaneously but disjointly.

\subsection{Investment certificate description and evaluation}

Investment certificates are a new class of investment and, for some aspects, similar to exchange traded funds (ETFs). Indeed, the two can provide passive investment strategies and can be traded on exchanges both by institutional investors and by retailers. Both ETFs and investment certificates can pay a coupon, either in the form of dividend or in the form of fixed amount subject to the achievement of certain conditions. Typically, ETFs do not have a maturity, while investment certificates do have. Moreover, the buyer of an investment certificate assumes the risk of default, but does not pay any management fee. Certificates, as well as ETFs, can have various types of underlying: corporate bonds, sovereign bonds, indexes, equities, currencies, commodities. Therefore, consequently, when investors trade certificates, they are also trading many risk sources, see Guillaume (2015), Chang et al. (2013), Chen (2003) and Viganò et al. (2019). Many certificates also include capital protection, and this can be exploited effectively in portfolio strategies, see Albeverio et al. (2017). A comprehensive classification of certificates, proposed by EUSIPA, divides certificates into four classes: capital protection, yield enhancement, participation and leverage products. Since the latter is an intrinsically speculative class, we focus our attention to one investment certificate for each of the other three classes that, moreover, were the most appreciated in the last years by investors ${ }^{2}$, and that can satisfy the protection purpose we are looking for. Thus, the three investment certificates that we consider are: the Uncapped Capital Protection (UCP) of the capital protection class, the Capped Bonus Certificates (CBC) in the yield enhancement class, and the Bonus Certificates (BC) in the participation class.

As previously mentioned, the pricing technique that we propose for highly structured products and, in particular, for investment certificate, needs to specify the map $f(\cdot)$ in (1)

$\overline{2 \text { See quarterly reports in EUSIPA }}$ webpage, www.eusipa.org/category/market-reports. 
Fig. 1 Payoff for the UCP investment certificate. Source: www.eusipa.org

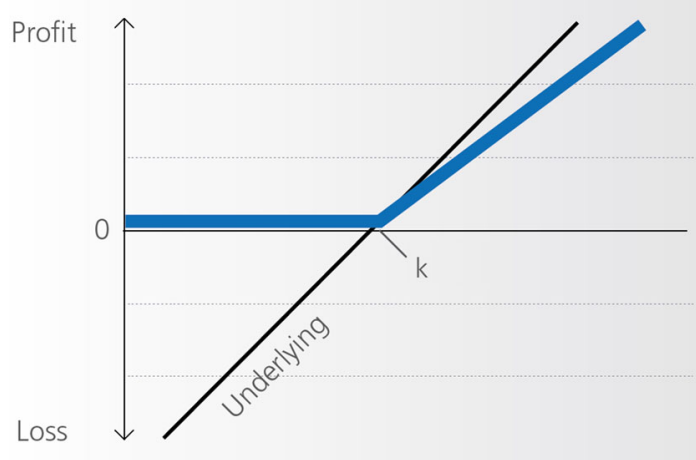

that characterizes the structure of the link between the value of the investment certificate at maturity and the return of its underlying.

Uncapped Capital Protection Let's consider first the UCP investment certificates, its payoff is depicted in Fig. 1.

This investment certificate provides a full protection of the nominal amount when the underlying value goes below a certain strike $K$ and follows the return of the underlying when its value goes above $K$. Then, assuming a nominal value of 1 and transforming the strike $K$ in the corresponding strike return $k$, to define the value of this contract in each node $\tilde{n} \in C(n)$ with $t(\tilde{n})-t(n)=m(i)$ we apply the following formula: ${ }^{3}$

$$
p_{i, \tilde{n}}= \begin{cases}1 & \text { if } \rho_{j, n, \tilde{n}} \leq k \\ 1+\rho_{j, n, \tilde{n}}, & \text { if } \rho_{j, n, \tilde{n}}>k\end{cases}
$$

Then, the fair current value of the instrument and its returns are computed applying (2) and (3).

Bonus Certificates Let's now consider the BC investment certificates, its payoff is depicted in Fig. 2.

This investment certificate provides a partial protection of the nominal amount when the underlying value is between a threshold $W$ and a strike $K$, while it follows the return of the underlying when its value goes below $W$ or above $K$. In case of protection, the final value of the instrument is set at $K$. Then, assuming a nominal value of 1 and transforming the amounts $W$ and $K$ in the corresponding returns $w$ and $k$, to define the value of this contract in each node $\tilde{n} \in C(n)$ with $t(\tilde{n})-t(n)=m(i)$ we apply the following formula:

$$
p_{i, \tilde{n}}= \begin{cases}1+k & \text { if } w \leq \rho_{j, n, \tilde{n}} \leq k \\ 1+\rho_{j, n, \tilde{n}}, & \text { if } \rho_{j, n, \tilde{n}}<w \vee \rho_{j, n, \tilde{n}}>k\end{cases}
$$

Then, the fair current value of the instrument and its returns are computed applying (2) and (3).

Capped Bonus Certificates Considering the CBC investment certificates, Fig. 3 shows its payoff.

3 The conversion from a strike price $K$ to its corresponding strike return $k$ is forced by the fact that the scenario tree contains the returns of the assets. If, e.g., the underlying value at the issue date is 15000 and the strike is set at $K=18000$, the corresponding return return is computed as $k=\frac{18000-15000}{15000}=0.2$. 
Fig. 2 Payoff for the BC investment certificate. Source: www.eusipa.org

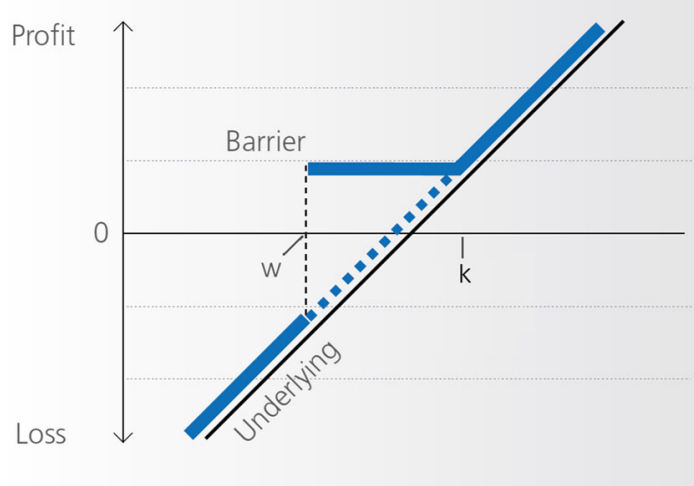

Fig. 3 Payoff for the CBC investment certificate. Source: www.eusipa.org

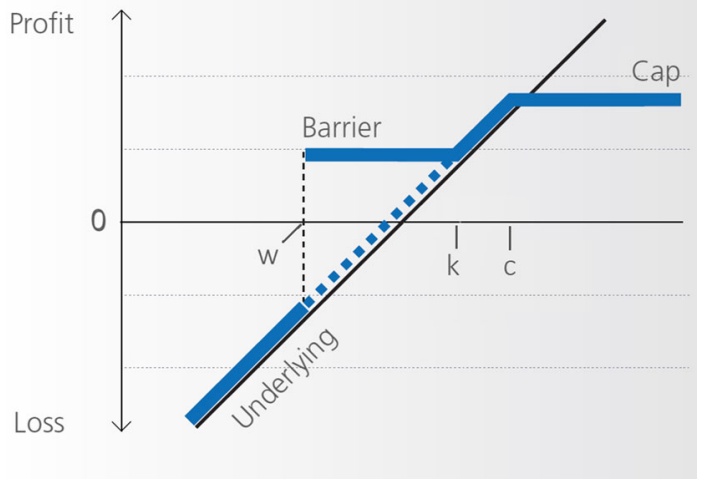

Th CBC investment certificate provides a partial protection as the $\mathrm{BC}$ investment certificate, but, moreover, when the value of the underlying exceeds an amount $C$, the return of the investment certificate is capped by the corresponding return $c$. Therefore, considering again a nominal value of 1 , to define the value of this contract in each node $\tilde{n} \in C(n)$ with $t(\tilde{n})-t(n)=m(i)$ we apply the following formula:

$$
p_{i, \tilde{n}}= \begin{cases}1+k & \text { if } w \leq \rho_{j, n, \tilde{n}} \leq k \\ 1+\rho_{j, n, \tilde{n}}, & \text { if } \rho_{j, n, \tilde{n}}<w \vee k<\rho_{j, n, \tilde{n}}<c \\ 1+c, & \text { if } \rho_{j, n, \tilde{n}} \geq c\end{cases}
$$

Then, the fair current value of the instrument and its returns are computed applying (2) and (3). In the market, most of CBC investment certificates are traded with $K=C$, then, we adopt the same structure in our empirical analysis and the pricing formula simplifies as follows:

$$
p_{i, \tilde{n}}= \begin{cases}1+\rho_{j, n, \tilde{n}}, & \text { if } \rho_{j, n, \tilde{n}}<w \\ 1+c, & \text { if } \rho_{j, n, \tilde{n}} \geq w\end{cases}
$$

Once the returns have been computed, the investment certificates and the put options are used in the model as any other asset. The pension fund manager can buy these instruments and income their value at maturity. An additional constraint for these assets is that their sale is 
Table 1 Asset universe adopted for the portfolio problem with asset, related asset type, corresponding Index, allocation upper bound and proportion of the initial portfolio used in the model

\begin{tabular}{llllll}
$i$ & Asset & Asset Type & Index & Upper bound & Initial Portfolio \\
\hline \hline 1 & Bank Account & Cash & EURIBOR 3-month & $30 \%$ & $15 \%$ \\
2 & 1-3 years & Treasury bond & Euro-Aggregate 1-3year & $6.25 \%$ \\
3 & 3-5 years & Treasury bond & Euro-Aggregate 3-5year & $6.25 \%$ \\
4 & 5-7 years & Treasury bond & Euro-Aggregate 5-7year & $6.25 \%$ \\
5 & 7-10 years & Treasury bond & Euro-Aggregate 7-10year & $6.25 \%$ \\
6 & 10+ years & Treasury bond & Euro-Aggregate 10+year & $100 \%$ \\
7 & Securitised & Securitised bond & Euro-Aggregate: Securitised & $6.25 \%$ \\
8 & Investment Grade & Corporate bond & Euro Corporate ex Subordinated 1\% Cap & $6.25 \%$ \\
9 & High Yield & Corporate bond & Euro HY B and above & $6.25 \%$ \\
10 & Real Estate & Real Estate & GPR General Europe & $20 \%$ & $6.25 \%$ \\
11 & MSCI & Public Equity & MSCI Europe & $50 \%$ & $10 \%$ \\
\hline
\end{tabular}

forbidden. This means that the pension fund manager cannot use the instruments to speculate and get an extra income by their intra-stage price change. In other words, the manager can use them only as hedging contracts, i.e. buying different levels of protection on a certain horizon against an unexpected fall of the underlying asset value.

\section{Asset universe, derivative universe and other settings}

The asset universe is the same considered in Moriggia et al. (2019) and includes 11 assets split in 4 classes: Cash, $i=1$; Bonds, $i=2, \ldots, 9$; Real Estate, $i=10$; Public Equity, $i=11$. The Treasury bonds are represented by five different maturity buckets. The Corporate bonds are subdivided into investment grade, i.e. rating higher than Baa3 Moody's or BBBStandard\&Poor's, and high yield, i.e. rating in the interval (Ba1, B3) Moody's or (BB+, B-) Standard\&Poor's, see (Bertocchi et al. 2013 [Chapter 5]). The list of all assets with the corresponding upper bounds is reported in Table 1. We also include the derivatives in the form of put options on Real Estate and on Public Equity. We consider put options with 1-year and with 2 -year maturity and with strike at $0 \%$, i.e. they protect from any possible loss on the underlying. For the purpose of this analysis, we add the investment certificates introduced in Sect. 2.2 written on Real Estate or on Public Equity. Thus, the investment certificates are of the three types: UCP, BC and CBC. Each investment certificates is considered with either 1-year or 2-year maturity. The maturities are chosen according to the tree discretization and to the market liquidity. The inclusion of investment certificates is tested with different combinations of the values of $w, k=c$. In particular, the value of $w$ is tested at either $-10 \%$ or $-5 \%$ combined with the values of $k$ (and $c$ for the $\mathrm{CBC}$ ) at $5 \%$ or at $10 \%$. Each derivative has an upper bound of 5\% in terms of allocation, and the whole class of derivative has a total upper bound of $20 \%$. To summarize, we consider the four put options and the 12 investment certificates reported in Table 2 .

\subsection{Other parameters and objective function}

The scenario tree is constructed with a final horizon $t_{H}=20$ years and we account five decisional stages at time $1,2,3,5$ and 10 , while the intermediate stages are every year. The branching structure of the stochastic tree (also called bushiness) is 8-4-2-2-2-2, i.e. the root node has 8 children, each of them has 4 children, etc. Then, the tree is composed of 512 scenarios which grow over the 7 stages. Such choice is consistent with the number of 
Table 2 Derivative universe adopted for the portfolio problem with asset type, corresponding underlying, strike $w$, barrier/cap $k=c$, allocation upper bound and proportion of the initial portfolio used in the model

\begin{tabular}{|c|c|c|c|c|c|c|c|}
\hline$i$ & Asset type & Maturity & Underlying & $w$ & $k=c$ & Upper bound & Initial Portfolio \\
\hline 12 & Put & 1-year & Real Estate & $0 \%$ & n.a. & ) & ) \\
\hline 13 & Put & 2-year & Real Estate & $0 \%$ & n.a. & & \\
\hline 14 & Put & 1-year & Public Equity & $0 \%$ & n.a. & & \\
\hline 15 & Put & 2-year & Public Equity & $0 \%$ & n.a. & & \\
\hline 16 & UCP & 1-year & Real Estate & ) & ) & & \\
\hline 17 & UCP & 2-year & Real Estate & & & & \\
\hline 18 & UCP & 1-year & Public Equity & & & & \\
\hline 19 & UCP & 2-year & Public Equity & & & $5 \%$ & $0 \%$ \\
\hline 20 & $\mathrm{BC}$ & 1-year & Real Estate & & & $5 \%$ & $0 \%$ \\
\hline 21 & $\mathrm{BC}$ & 2-year & Real Estate & & & & \\
\hline 22 & $\mathrm{BC}$ & 1-year & Public Equity & $-10 \% /-5 \%$ & $5 \% / 10 \%$ & & \\
\hline 23 & $\mathrm{BC}$ & 2-year & Public Equity & & & & \\
\hline 24 & $\mathrm{CBC}$ & 1-year & Real Estate & & & & \\
\hline 25 & $\mathrm{CBC}$ & 2-year & Real Estate & & & & \\
\hline 26 & $\mathrm{CBC}$ & 1-year & Public Equity & & & & \\
\hline 27 & $\mathrm{CBC}$ & 2-year & Public Equity & & & & \\
\hline
\end{tabular}

scenarios adopted in practice by an insurance company that manages a pension fund and, thus, it is considered as representative of the degree of uncertainty faced by the portfolio manager, see Consigli et al. (2017). Comparing to that choice, we reduce the branching in the first stage to compensate the complexity induced by the stochastic dominance constraints.

In the objective function (4), we consider four target variables $Y_{a, n}$ :

$Y_{1, n}:$ a joint measure of the ALM risk and of the liquidity gap,

$Y_{2, n}$ : a measure of the return adjusted by the risk,

$Y_{3, n}$ : the cumulative sponsor contribution,

$Y_{4, n}$ : the difference between the Defined Benefit Obligation and the portfolio value.

The stages in which we account these variables are $\tau_{1}=1, \tau_{2}=3, \tau_{3}=10, \tau_{4}=20$ and we assign the weights $\lambda_{1}=10 \%, \lambda_{2}=30 \%, \lambda_{3}=40 \%$ and $\lambda_{4}=20 \%$.

When active, the SD constraints are imposed on the stages with $t=3$ and $t=10$. Indeed, our purpose is to test the quality of the solution with respect to a benchmark at an intermediate stage and in the long-term. For the long-term we do not apply SD in $t=20$ because, on the one hand, the results are very similar to the case when SD is imposed at $t=10$, and, on the other hand, to impose the SD at $t=20$ would induce too much computational complexity making the model intractable, especially in the FSD case. For the SD, the benchmark is created assuming as reference portfolio the strategy given by the initial portfolio, see Table 1 and Table 2. Indeed, the initial portfolio is the current strategy of the pension fund and, thus, it represents the investment that the pension fund manager considers to be the best. Therefore, we observe the evolution of this portfolio over the scenario tree imposing a fix-rebalancing in each decisional node to restore the initial proportions among the assets. We remark that in the initial portfolio there are not derivatives because we want to observe and quantify the benefit given by their inclusion.

\section{Empirical results}

In this Section, we perform several analysis to understand whether the investment certificates could improve the performance of the pension fund and efficiently substitute the put options as hedging instruments. 


\subsection{Stochastic dominance and derivatives}

We analyse and compare the characteristics of the solutions applying the different definitions of stochastic dominance and including in the asset universe either all the derivatives or only the investment certificates. Figure 4 shows the first set of results. In particular, we represent the optimal here-and-now $(\mathrm{H} \& \mathrm{~N})$ solution, i.e. the optimal portfolio in the root node, obtained under different combination of constraints and derivative universe. Moreover, for each solution, we report the statistics of the distribution of the wealth of the portfolio in the stage $t=10$ where, when imposed, the SD constraints have a more relevant impact. Finally, for each case, we show the value of the objective function that summarizes the expected shortfall of the variables mentioned in the previous section. The investment certificates considered in these results are with $w=-5 \%$ and $k=c=5 \%$. The first result on the left is the unprotected optimal portfolio that corresponds to the case in which neither the SD constraints nor the derivatives are included in the model. Therefore, it represents the solution we obtain with the original model. Even if the portfolio wealth is not a target of the model, we compare the statistics of this variable at $t=10$. Moving to the right of Fig. 4, we find the two cases in which the derivatives are included while the SD constraints are not. In the case, where the derivatives are also available, we notice that the objective value benefits remarkably by their protection $(26,973$ vs 34,616$)$. Indeed, both the two put options with 1-year maturity are used in the H\&N solution touching the upper bound of 5\% and only a small portion, the $1 \%$, is allocated in the $\mathrm{BC}$ on Public Equity. The wealth distribution is slightly worst than the unprotected case because the protection ensured by the derivatives has a cost, but the expected shortfalls of the variables in the objective function decrease as remarked by the decreasing objective value. In particular, the put options give a huge protection but their cost is quite relevant. In the trade-off protection/cost, the optimal solution considers the former more relevant. However, when only the investment certificates are available, it is still possible to improve the objective value $(32,599$ vs 34,615$)$ investing the $4.8 \%$ of the H\&N portfolio in the BC on Real Estate. Moreover, the wealth distribution is slightly better than in the case with put options. This means that the investment certificates give a more limited protection but at a lower cost.

Let's introduce now the benchmark in the model and let's implement the SD constraints. As we can notice, the $H \& N$ portfolio of the benchmark is very aggressive. The $60 \%$ of the portfolio is allocated in the two most risky assets. Indeed, the unprotected portfolio, i.e. the solution with the original model, already moved the portfolio to a much more safety assets. However, such aggressive portfolio is able to generate wealth distributions at $t=3$ and, more relevant, at $t=10$ that have a higher mean value than the unprotected portfolio, even if the tail indicators are worse. Therefore, let's assume that the pension fund manager would like to account not only for the variables in the objective function, but also would like to have an optimal portfolio that generates a wealth distribution better, in the SD sense, than the benchmark.

The further two results on the right of Fig. 4 show the optimal solution when the FSD with respect to the benchmark wealth distribution are active at $t=3$ and $t=10$. As we mention above, these solutions are obtained through a mixed-integer problem and, to be able to solve it within few hours, we had to set the relative gap at $15 \%$. However, it is possible to find a portfolio whose wealth distribution dominates the wealth distribution of the benchmark in the FSD sense. Indeed, all the statistics of the wealth distribution at $t=10$ are better: the averages are 532,952 and 537,346 vs 500,657 , the V@Rs are 278,933 and 286,454 vs 238,172 , the AV@Rs are 255,331 and 261,606 vs 199,244. Clearly, to satisfy the FSD constraints the objective function gets remarkably worse than the cases without the SD. This worsening is 


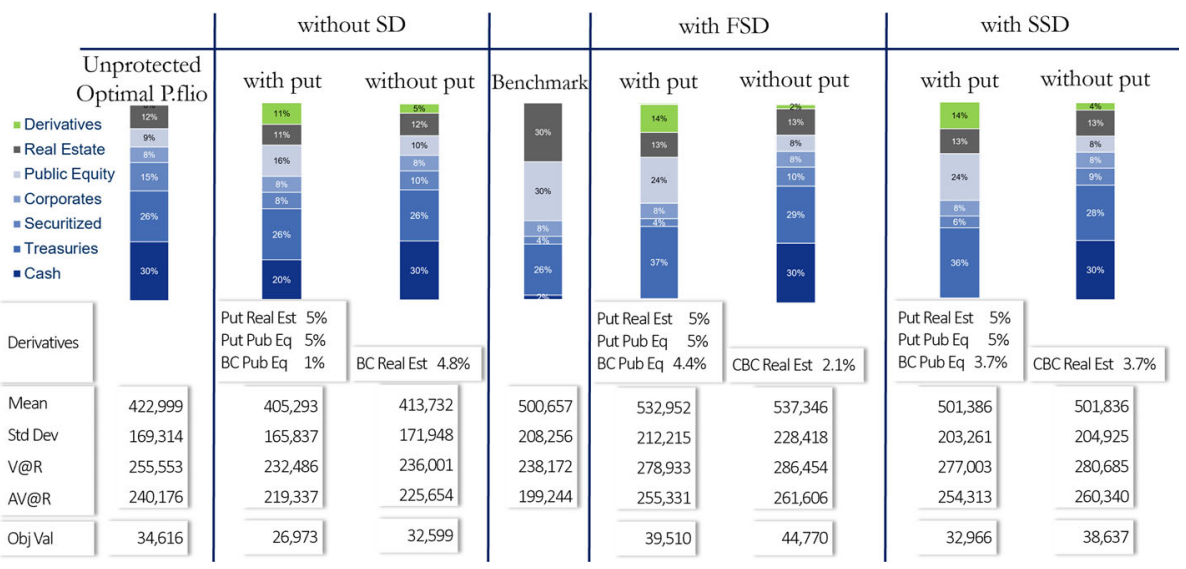

Fig. 4 Optimal solution obtained solving the unprotected model (without SD and without derivatives), solving the model with and without FSD, with and without SSD. Each case with and without put options. We present the $H \& \mathrm{~N}$ portfolios, the detail of the allocation in derivatives, the statistics of the wealth distribution at $t=10$ and the value of the objective function. The investment certificates are with $w=-5 \%$ and $k=c=5 \%$

the cost to satisfy the FSD constraints. Thus, the pension fund manager can choose whether it is an acceptable cost or not. The differences between the portfolio with or without put options are similar to the ones highlighted for the case without SD: the put options provide more protection at a higher cost. This effect is clear comparing the statistics of the wealth distribution and the objective functions.

The last two results on the right hand side of Fig. 4 show the optimal solution when the SSD are imposed at $t=3$ and $t=10$. Again, all the statistics of the wealth distribution at $t=10$ are better than the benchmark: the averages are 501,386 and 501,836 vs 500,657, the V@Rs are 277,003 and 280,685 vs 238,172, the AV@Rs are 254,313 and 260,340 vs 199,244 . As in the FSD case, also using the SSD constraints the objective function gets slightly worse. And the differences between the portfolio with or without put options remark again that the put options provide better protection but at a higher cost.

We can conclude that the SSD is somehow in the middle between the case without SD and the FSD case: it is possible to dominate the benchmark, but the optimal objective value is still close to the case without SD.

In Fig. 5, we show the quantile function of the wealth for the three cases that include put options: the case without SD, the case with FSD, and the case with SSD. We remark that, comparing the quantile function of the optimal portfolio and of the benchmark, we are basically checking the presence of FSD. We can notice that in the case without SD and in the case with SSD, the FSD is not imposed, and, thus, achieved neither at $t=3$ nor at $t=10$. In particular, the imposition of the SSD makes the two distribution closer but not enough to guarantee the FSD, while in the case without SD the quantile function of the optimal portfolio wealth is remarkably worst than the benchmark. Considering the FSD case, we observe that constraints (5) are active in particular in $t=10$, meaning that to impose the FSD in $t=10$ basically implies that the FSD is achieved also in $t=3$, implicitly.

In Fig. 6, we show the integrated quantile function of the wealth for the three cases that include put options: the case without SD, the case with FSD, and the case with SSD. We remark that, comparing the integrated quantile function of the optimal portfolio and of the benchmark, we are basically checking the presence of SSD. We notice that, in the case with FSD, the SSD is automatically implied as it is described in Sect. 2.1. In the case without SD, 
without SD

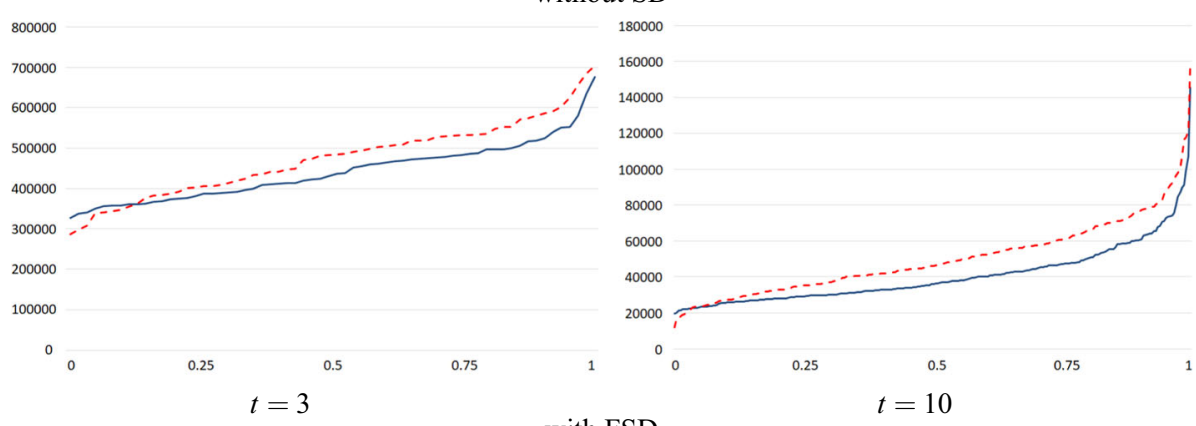

with FSD

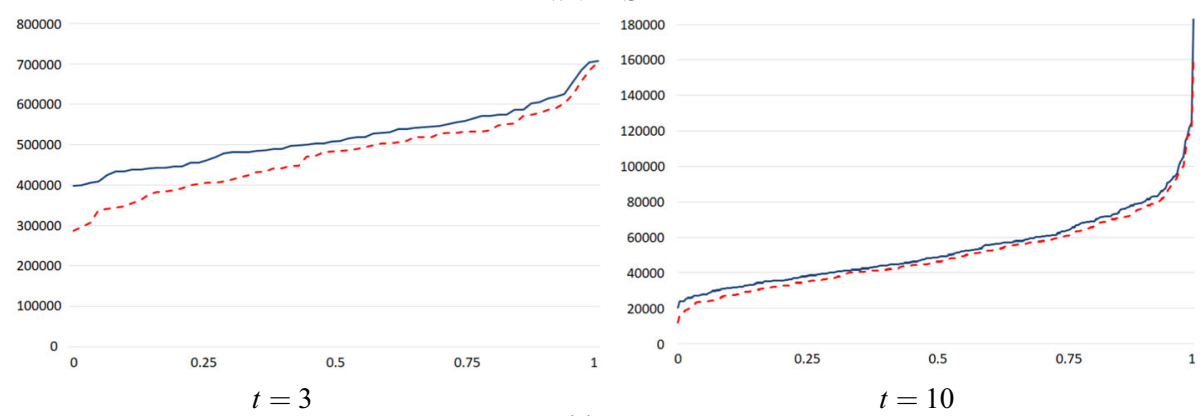

with SSD

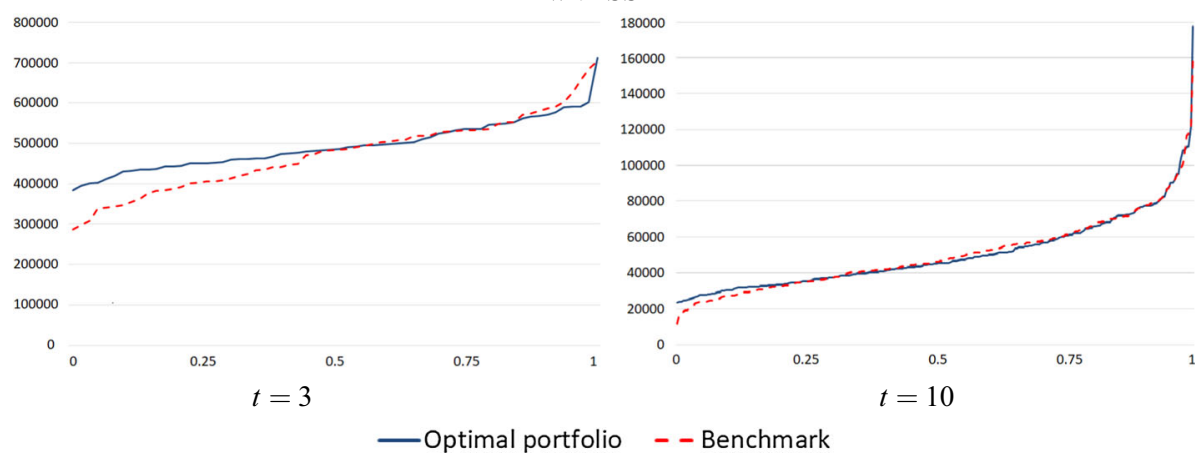

Fig. 5 Quantile function of the wealth for the cases without SD, with FSD, and with SSD, all with put options. We consider the wealth distributions at $t=3$ and at $t=10$. The solid line represents the wealth of the optimal portfolio, the dashed line represents the wealth of the benchmark

the SSD is not achieved. Considering the SSD case, we observe that constraints (5) are active for almost all the nodes in $t=10$, while in $t=3$ it is active only in the very left part of the distribution meaning that the SSD constraints in $t=3$ have an impact on the solution, even if not very relevant.

All the results shown above are obtained by implementing and solving a linear programming model with CPLEX 12.6.0.1 in GAMS 24.3.3, with an Intel(R) Core(TM) i7-8650U CPU 1.90GHz with 16GB RAM running Windows 10. All the input for the optimization are computed in MATLAB R2019b. CPLEX generates the original model with 1388426 single equations, 1525955 single variables, 5736390 non zero elements and generates the model with stochastic dominance constraints with 1389386 single equations, 1595587 single vari- 

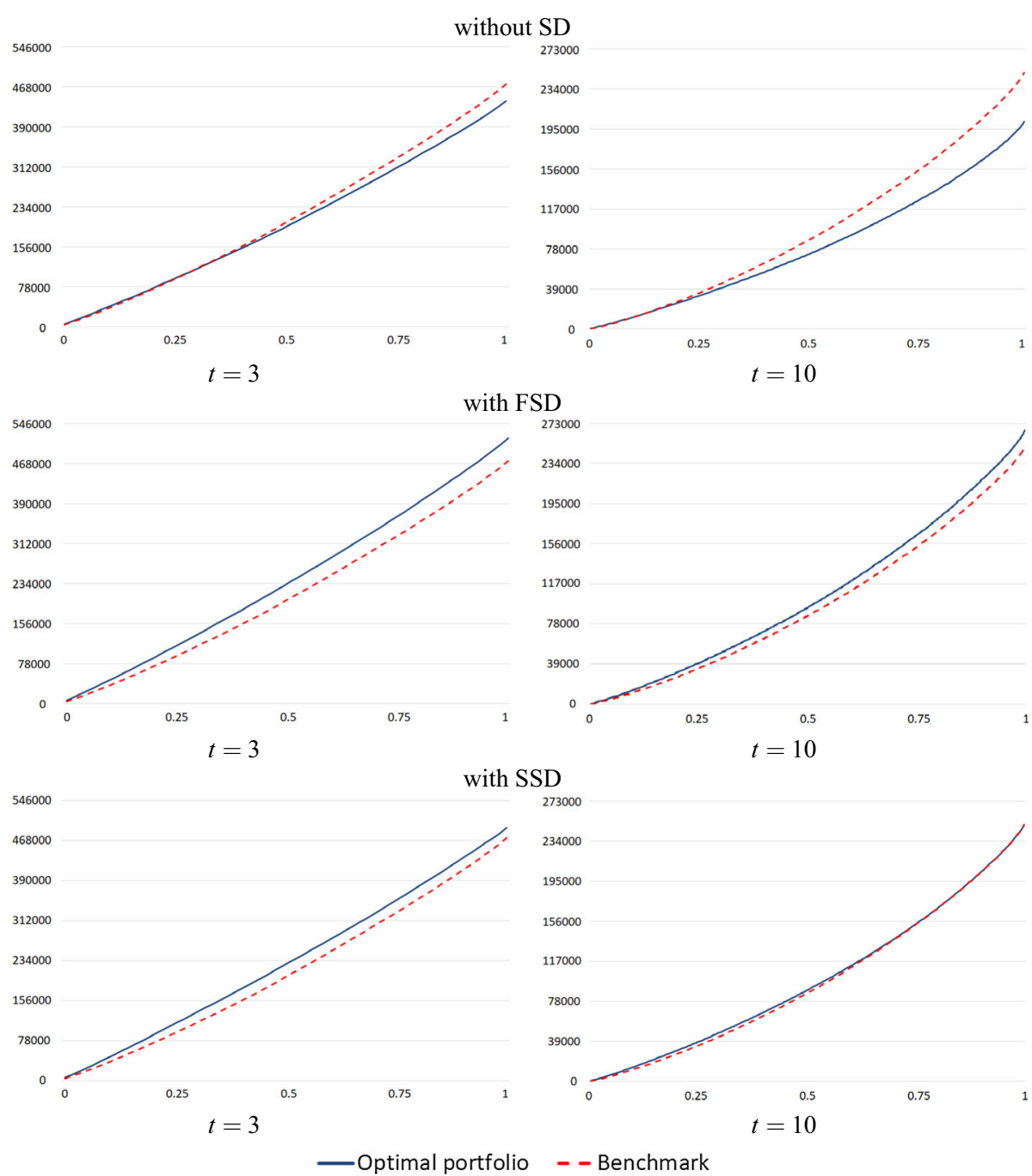

Fig. 6 Integrated quantile function of the wealth for the cases without SD, with FSD, and with SSD, all with put options. We consider the wealth distributions at $t=3$ and at $t=10$. The solid line represents the wealth of the optimal portfolio, the dashed line represents the wealth of the benchmark

ables, 5945606 non zero elements. Then, the FSD case has 69632 binary variables and the CPLEX solver was not able to reach the $10 \%$ of relative gap in 24 hours, so the relative gap has been set at $15 \%$ and the solver found a solution within 2 hours. The SSD case takes approximately 30 minutes to solve, the case without SD approximately 10 minutes. Problem instances with larger dimension gave similar results but inducing memory issues.

\subsection{Sensitivity to the parameters of the investment certificates}

Let's now consider in the derivative universe other investment certificates having different combinations of the parameters $w, k$ and $c$. We perform this sensitivity analysis considering 
only the case without SD and with SSD. We do not include the FSD because their computational burden and because in the few tests that we did their results are similar to the SSD case. Moreover, we do not display the evidences in terms of wealth distribution and objective value because the comments would be aligned to the ones already deeply analysed in Fig. 4. Therefore, in Table 3, we compare types and quantities of hedging assets in the H\&N portfolio. We can notice that the investment certificates are never preferred to the put options. Indeed, when the asset universe includes put options, these assets absorb all the allocation available (5\%). However, the investment certificates are not dominated by the put options and they represent a complementary asset to mitigate the risk at a relatively lower cost. Moreover, the amount allocated in investment certificates tends to increase as the values of $w$ and $k=c$ diverge, i.e. when they diversify from the behaviour both of the put options and of the underlying itself. Similarly, the investment certificates appear to be more attractive when the SSD constraints are imposed. However, among the 12 available investment certificates, only a very few are used in the H\&N solutions, and also in subsequent stages. These are: the CBC on Real Estate, the BC on Public Equity and the UCP either on Real Estate or on Public Equity. Only in few cases the investment certificates are combined with each other. Moreover, when the put options are not available, the solution tends to disinvest in Real Estate and Public Equity to move to safer assets. Then, the turnover flexibility is mainly exploited for this fly-to-safety, and the residual risky investments requires less hedging with investment certificates.

Both the options and the investment certificates adopted in all the $\mathrm{H} \& \mathrm{~N}$ portfolios described above have 1-year maturity. Moreover, also in the subsequent stages, the portfolio never uses 2-year maturity derivatives. This is consistent with what already found in Moriggia et al. (2019) where the put options with 2-year maturity were never used in the optimal portfolio. Indeed, since the selling of these instruments is forbidden, the optimal portfolio prefers to remain flexible and to buy only 1-year derivatives and, in case the protection is needed also for the next year, to buy again another 1-year maturity derivative. Such results could change in case of high transaction costs which are not included in this model since, generally, a large pension fund pays a relatively small amount of transaction fees.

\subsection{Stability of the H\&N optimal portfolios}

All the results shown in previous sections were computed for a single multistage stochastic scenario tree to be able to compare the different cases in a unique stochastic framework. However, once a new stochastic scenario tree is generated, running again the solver could lead to a different optimal solution. In this section, we want to observe the stability of the solution with respect to the stochastic scenario tree. Therefore, we generate 20 different stochastic scenario trees and we solve the ALM model considering the case with all the derivatives and imposing the SSD constraints.

In Table 4, we report the summary of the H\&N allocations of the 20 solutions. In particular, we show the minimum allocation in each asset, the average allocation, and the maximum allocation. We notice that the portfolio never invest in Cash and that the allocation in Corporates is always the same. The allocation in Treasury and Securitised is quite different among the 20 portfolios, while the allocation in Public Equity and Real Estate varies, but it is more stable. We decide to split the information about the derivatives to highlight that the investment in Put Options is constant and touches always the upper bound of $10 \%$, while the allocation in Investment Certificates could change relevantly. In particular, the Investment Certificates used across the 20 portfolios are mainly three: the BC 1-year on Public Equity, the UCP 1-year on Public Equity, the CBC 1-year on Public Equity. 


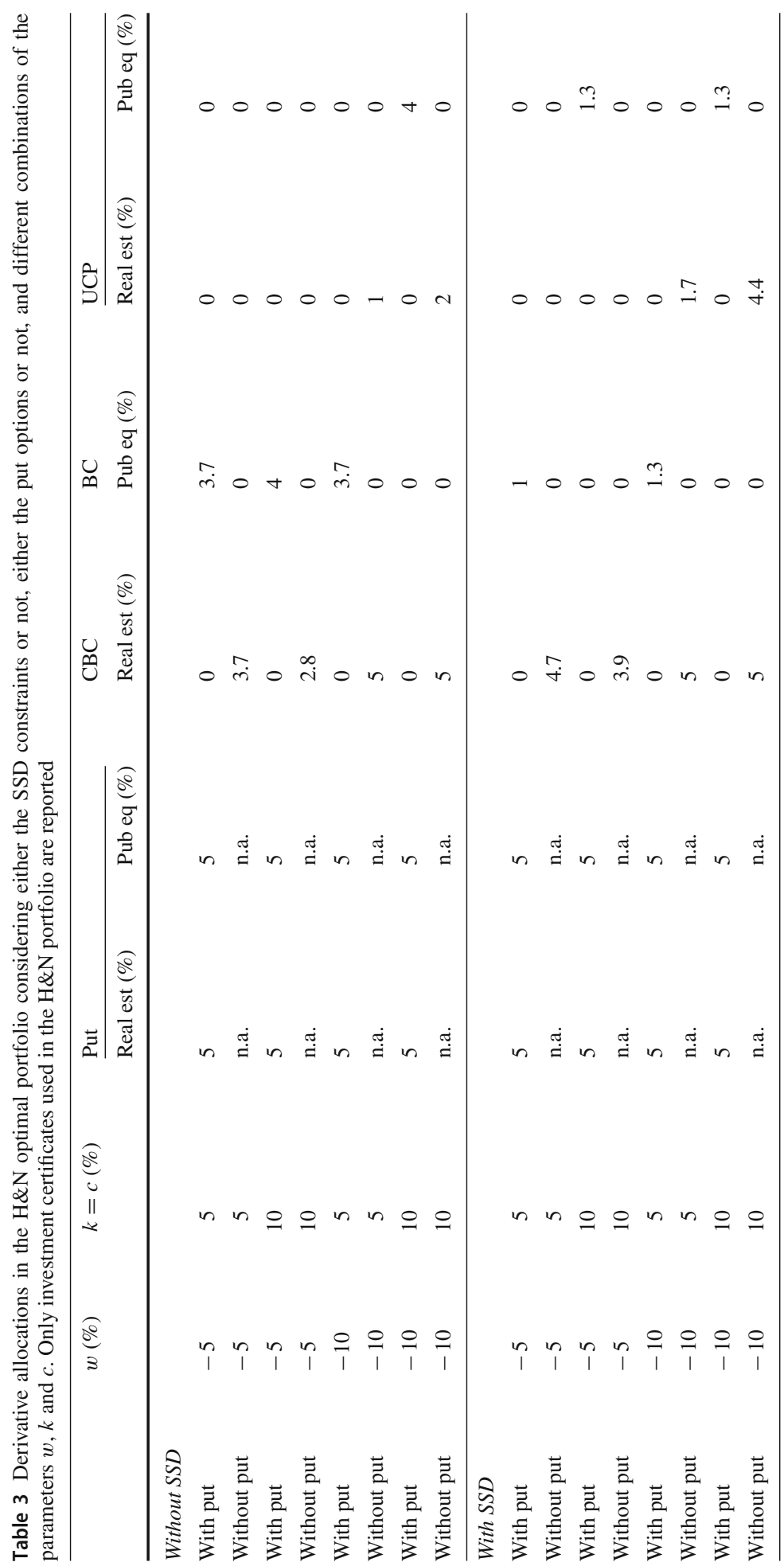


Table 4 Distribution of the allocation of the $\mathrm{H} \& \mathrm{~N}$ portfolios for the 20 representative solutions

Table 5 Distribution of the main statistics on the wealth at $t=10$ for the 20 representative solutions

\begin{tabular}{llll}
\hline Asset class & Min $(\%)$ & Average (\%) & Max (\%) \\
\hline Cash & - & 0 & - \\
Treasury & 33.2 & 34.9 & 36.9 \\
Securitised & 5.9 & 7.0 & 9.1 \\
Corporates & - & 8 & - \\
Public equity & 12.1 & 13.0 & 13.8 \\
Real estate & 22.8 & 23.5 & 24.4 \\
Put options & - & 10 & - \\
Investment certificates & 1.4 & 3.6 & 4.8 \\
\hline
\end{tabular}

\begin{tabular}{llll}
\hline Statistic & Min & Average & Max \\
\hline Mean & 497,586 & 501,010 & 504,296 \\
SD & 198,850 & 203,079 & 205,759 \\
V@R & 268,165 & 275,249 & 279,319 \\
AV@R & 244,414 & 252,953 & 258,306 \\
\hline
\end{tabular}

In Table 5, we report the summary of the statistics regarding the wealth distribution at $t=10$ achieved by the optimal portfolios in the 20 representative cases. We notice that all the statistics are somehow stable and among the 20 portfolios and the 20 stochastic tree we do not observe any extreme situation. This is due both to a stability of the stochastic scenario tree generation technique and to the stability of the solution given by the ALM model. Indeed, the scenario generation algorithm never produces extreme trajectories, and the ALM model is able to face and manage also very volatile scenarios.

\section{Conclusion}

The paper proposes an extension of the Asset Liability Management model presented in Consigli et al. (2017) and Moriggia et al. (2019). In particular, we suggest a technique to define the value and the returns of highly structured products on a discrete scenario tree, and we test the inclusion of investment certificates in a pension fund portfolio. These instruments are analysed with respect to plain vanilla put options and in presence of FSD and SSD constraints with respect to a benchmark portfolio. The empirical results show that the put options are more effective to hedge the portfolio than the investment certificates. However, the investment certificates still play a role to create the optimal portfolio and are used both in the $\mathrm{H} \& \mathrm{~N}$ solution and in the dynamic strategy. As far as the investment certificate features are concerned, considering that we need to keep the model computationally tractable, it was possible to test disjointly different payoff structures and two distinct maturities maintaining the number of available assets below 27. On the one hand, the limit in the maturities did not affect the solution since only 1-year maturities are actually used in the portfolio; on the other hand, a wider selection of available investment certificates could prejudice the tractability of the model. The issue could be addressed in a further work by considering the parameters $w$, $k$ and $c$ to be decisional variable. 
Funding Open access funding provided by Università degli Studi di Bergamo within the CRUI-CARE Agreement.

Open Access This article is licensed under a Creative Commons Attribution 4.0 International License, which permits use, sharing, adaptation, distribution and reproduction in any medium or format, as long as you give appropriate credit to the original author(s) and the source, provide a link to the Creative Commons licence, and indicate if changes were made. The images or other third party material in this article are included in the article's Creative Commons licence, unless indicated otherwise in a credit line to the material. If material is not included in the article's Creative Commons licence and your intended use is not permitted by statutory regulation or exceeds the permitted use, you will need to obtain permission directly from the copyright holder. To view a copy of this licence, visit http://creativecommons.org/licenses/by/4.0/.

\section{References}

Albeverio, S., Steblovskaya, V., \& Wallbaum, K. (2017). The volatility target effect in structured investment products with capital protection. Review of Derivatives Research pp. 1-29. https://doi.org/10.1007/ s11147--017--9138--2.

Bertocchi, M., Consigli, G., D’Ecclesia, R., Giacometti, R., Moriggia, V., \& Ortobelli, S. (2013). Euro bonds: Markets, infrastructure and trends. Singapore: World Scientific Books.

Bradley, S. P., \& Crane, D. B. (1972). A dynamic model for bond portfolio management. Management Science, 19(2), 139-151.

Bradley, S. P., \& Crane, D. B. (1980). Managing a bank bond portfolio over time. In M. A. H. Dempster (Ed.), Stochastic Programming (pp. 449-471). London: Academic Press.

Broeders, D., Chen, A., \& Koos, B. (2009). An institutional evaluation of pension funds and life insurance companies. Amsterdam: De Nederlandsche Bank.

Cariño, D. R., \& Ziemba, W. T. (1998a). Formulation of the Russell-Yasuda Kasai financial planning model. Operations Research, 46(4), 433-449.

Cariño, D. R., Kent, T., Myers, D. H., Stacy, C., Sylvanus, M., Turner, A. L., et al. (1994). The Russell-Yasuda Kasai model: An asset/liability model for a Japanese insurance company using multistage stochastic programming. Interfaces, 24(1), 29-49.

Cariño, D. R., Myers, D. H., \& Ziemba, W. T. (1998b). Concepts, technical issues, and uses of the RussellYasuda Kasai financial planning model. Operations Research, 46(4), 450-462.

Chang, E. C., Luo, X., Shi, L., \& Zhang, J. E. (2013). Is warrant really a derivative? Evidence from the chinese warrant market. Journal of Financial Markets, 16(1), 165-193.

Chen, S. Y. (2003). Valuation of covered warrant subject to default risk. Review of Pacific Basin Financial Markets and Policies, 6(01), 21-44.

Consigli, G. (2007). Individual asset liability management for individual investors. In S. A. Zenios \& W. T. Ziemba (Eds.), Handbook of Asset and Liability Management: Applications and case studies (pp. 752-827)., North-Holland Finance Handbook Series Amsterdam: Elsevier.

Consigli, G., \& Dempster, M. A. H. (1998a). The CALM stochastic programming model for dynamic assetliability management. Worldwide Asset and Liability Modeling, 10, 464.

Consigli, G., \& Dempster, M. A. H. (1998b). Dynamic stochastic programming for asset-liability management. Annals of Operations Research, 81, 131-162.

Consigli, G., \& Moriggia, V. (2014). Applying stochastic programming to insurance portfolios stress-testing. Quantitative Finance Letters, 2(1), 7-13.

Consigli, G., \& di Tria, M. (2012). Optimal long-term property and casualty ALM with risk capital control. Asset-Liability Management for Financial Institutions, 1, 137-150.

Consigli, G., di Tria, M., Gaffo, M., Iaquinta, G., Moriggia, V., \& Uristani, A. (2011). Dynamic portfolio management for property and casualty insurance. In Stochastic optimization methods in finance and energy, Springer, Berlin, pp. 99-124.

Consigli, G., Iaquinta, G., Moriggia, V., di Tria, M., \& Musitelli, D. (2012). Retirement planning in individual asset-liability management. IMA Journal of Management Mathematics, 23(4), 365-396.

Consigli, G., Moriggia, V., Benincasa, E., Landoni, G., Petronio, F., Vitali, S., et al. (2017). Optimal multistage defined-benefit pension fund management. In G. Consigli, S. Stefani, \& G. Zambruno (Eds.), Recent advances in commmodity and financial modeling: quantitative methods in banking, finance, insurance, energy and commodity markets. Berlin: Springers International Series in Operations Research and Management Science. 
Consigli, G., Moriggia, V., \& Vitali, S. (2019). Long-term individual financial planning under stochastic dominance constraints. Annals of Operations Research, 292, 973-1000.

Davis, P. E. (2000). Portfolio regulation of life insurance companies and pension funds. Economics.

Dempster, M.A.H., \& Ireland, A.M. (1988). MIDAS: An expert debt management advisory system. In Data, expert knowledge and decisions (pp. 116-127). Berlin: Springer.

Dempster, MAH.,\& Ireland, AM. (1989). Object-oriented model integration in MIDAS [Manager's Intelligent Debt Advisory System]. In Proceedings of the twenty-second annual hawaii international conference on system sciences, 1989. Vol. III: Decision support and knowledge based systems track, IEEE (Vol. 3, pp. 612-620).

Dempster, M. A. H., \& Ireland, A. M. (1991). Object-oriented model integration in a financial decision support system. Decision Support Systems, 7(4), 329-340.

Dempster, M. A. H., Germano, M., Medova, E. A., \& Villaverde, M. (2003). Global asset liability management. British Actuarial Journal, 9(01), 137-195.

Dentcheva, D., \& Ruszczynski, A. (2003). Optimization with stochastic dominance constraints. SIAM Journal on Optimization, 14(2), 548-566.

Dentcheva, D., \& Ruszczyński, A. (2004). Semi-infinite probabilistic optimization: First-order stochastic dominance constrain. Optimization, 53(5-6), 583-601.

Dert, C. L. (1998). A dynamic model for asset liability management for defined benefit pension funds. Worldwide asset and liability modeling, 10, 501-536.

Devolder, P., Levantesi, S., \& Menzietti, M. (2020). Automatic balance mechanisms for notional defined contribution pension systems guaranteeing social adequacy and financial sustainability: An application to the italian pension system. Annals of Operations Research. https://doi.org/10.1007/s10479-020-03819$\mathrm{x}$.

Dupačová, J., \& Kopa, M. (2012). Robustness in stochastic programs with risk constraints. Annals of Operations Research, 200(1), 55-74.

Dupačová, J., \& Kopa, M. (2014). Robustness of optimal portfolios under risk and stochastic dominance constraints. European Journal of Operational Research, 234(2), 434-441.

Geyer, A., \& Ziemba, W. T. (2008). The Innovest Austrian pension fund financial planning model InnoALM. Operations Research, 56(4), 797-810.

Gollier, C. (2008). Intergenerational risk-sharing and risk-taking of a pension fund. Journal of Public Economics, 92(5), 1463-1485.

Guillaume, T. (2015). Autocallable structured products. Journal of Derivatives, 22(3), 73.

Guiso, L., Haliassos, M., \& Jappelli, T. (2002). Household portfolios. Cambridge: MIT Press.

Hadar, J., \& Russell, W. R. (1969). Rules for ordering uncertain prospects. The American Economic Review, $59(1), 25-34$.

Hanoch, G., \& Levy, H. (1969). The efficiency analysis of choices involving risk. The Review of Economic Studies, 36, 335-346.

Hardy, M. (2003). Investment guarantees: modeling and risk management for equity-linked life insurance (Vol. 215). New York: Wiley.

Kopa, M., \& Post, T. (2015). A general test for ssd portfolio efficiency. OR Spectrum, 37(3), 703-734.

Kopa, M., \& Rusỳ, T. (2020). A decision-dependent randomness stochastic program for asset-liability management model with a pricing decision. Annals of Operations Research. https://doi.org/10.1007/s10479020-03583-y.

Kopa, M., Moriggia, V., \& Vitali, S. (2018). Individual optimal pension allocation under stochastic dominance constraints. Annals of Operations Research, 260(1-2), 255-291.

Kuosmanen, T. (2004). Efficient diversification according to stochastic dominance criteria. Management Science, 50(10), 1390-1406.

Kusy, M. I., \& Ziemba, W. T. (1986). A bank asset and liability management model. Operations Research, 34(3), 356-376.

Luedtke, J. (2008). New formulations for optimization under stochastic dominance constraints. SIAM Journal on Optimization, 19(3), 1433-1450.

McKendall, R. Zenios, S.A., \& Holmer, M. (1994). Stochastic programming models for portfolio optimization with mortgage backed securities: Comprehensive research guide. In Operations research models in quantitative finance, Springer, Berlin, pp. 134-171

Moriggia, V., Kopa, M., \& Vitali, S. (2019). Pension fund management with hedging derivatives, stochastic dominance and nodal contamination. Omega, 87, 127-141.

Mulvey, J. M. (1994a). An asset-liability investment system. Interfaces, 24(3), 22-33.

Mulvey, J.M. (1994b). Financial planning via multi-stage stochastic programs. Mathematical Programming: State of the Art. 
Mulvey, J. M., Gould, G., \& Morgan, C. (2000). An asset and liability management system for Towers PerrinTillinghast. Interfaces, 30(1), 96-114.

Mulvey, J. M., Simsek, K. D., \& Zhang, Z. (2006). Improving investment performance for pension plans. Journal of Asset Management, 7(2), 93-108.

Mulvey, J. M., Ural, C., \& Zhang, Z. (2007). Improving performance for long-term investors: wide diversification, leverage, and overlay strategies. Quantitative Finance, 7(2), 175-187.

Mulvey, J. M., Simsek, K. D., Zhang, Z., Fabozzi, F. J., \& Pauling, W. R. (2008). Assisting defined-benefit pension plans. Operations Research, 56(5), 1066-1078.

Nielsen, S. S., \& Zenios, S. A. (1996). A stochastic programming model for funding single premium deferred annuities. Mathematical Programming, 75(2), 177-200.

Pflug, G. C., \& Swietanowski, A. (1999). Dynamic asset allocation under uncertainty for pension fund management. Control and Cybernetics, 28, 755-777.

Post, T. (2003). Empirical tests for stochastic dominance efficiency. The Journal of Finance, 58(5), 1905-1931.

Post, T., \& Kopa, M. (2017). Portfolio choice based on third-degree stochastic dominance. Management Science, 63(10), 3381-3392.

Post, T., Fang, Y., \& Kopa, M. (2015). Linear tests for decreasing absolute risk aversion stochastic dominance. Management Science, 61(7), 1615-1629.

Quirk, J. P., \& Saposnik, R. (1962). Admissibility and measurable utility functions. The Review of Economic Studies, 29, 140-146.

Viganò, B., Vitali, S., Moriggia, V., \& Zanotti, G. (2019). The investment certificates in the Italian market: A comparison of quoted and estimated prices. Journal of Financial Management, Markets and Institutions, 7(2), 1950002.

Vitali, S., Moriggia, V., \& Kopa, M. (2017). Optimal pension fund composition for an italian private pension plan sponsor. Computational Management Science, 14(1), 135-160.

Yang, X., Gondzio, J., \& Grothey, A. (2010). Asset liability management modelling with risk control by stochastic dominance. Journal of Asset Management, 11(2), 73-93.

Zenios, S. A. (1995). Asset/liability management under uncertainty for fixed-income securities. Annals of Operations Research, 59(1), 77-97.

Zenios, S. A., \& Ziemba, W. T. (2006). Handbook of asset and liability management: Theory and methodology (Vol. 1)., North-Holland Finance Handbook Series Amsterdam: Elsevier.

Zenios, S. A., \& Ziemba, W. T. (2007). Handbook of Asset and Liability Management: Applications and case studies (Vol. 2)., North-Holland Finance Handbook Series Amsterdam: Elsevier.

Ziemba, W. T. (2007). The Russell-Yasuda, InnoALM and related models for pensions, insurance companies and high net worth individuals. In S. A. Zenios \& W. T. Ziemba (Eds.), Handbook of asset and liability management: Applications and case studies (pp. 861-962)., North-Holland Finance Handbook Series Amsterdam: Elsevier.

Ziemba, W. T., \& Mulvey, J. M. (Eds.). (1998). Worldwide asset and liability modeling (Vol. 10). Cambridge: Cambridge University Press.

Publisher's Note Springer Nature remains neutral with regard to jurisdictional claims in published maps and institutional affiliations. 